\title{
Filigrane
}

Écoutes psychothérapiques

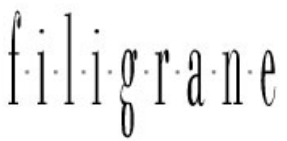

\section{Oedipe roi et la langue de Sophocle}

\section{Patrick Mahony}

Volume 20, numéro 2, automne 2011

La langue vivante de la clinique psychanalytique II

URI : https://id.erudit.org/iderudit/1007610ar

DOI : https://doi.org/10.7202/1007610ar

Aller au sommaire du numéro

\section{Éditeur(s)}

Revue Santé mentale au Québec

ISSN

1192-1412 (imprimé)

1911-4656 (numérique)

Découvrir la revue

\section{Citer cet article}

Mahony, P. (2011). Oedipe roi et la langue de Sophocle. Filigrane, 20(2), 33-52. https://doi.org/10.7202/1007610ar

\section{Résumé de l'article}

Ce classique de Sophocle, qui continue à nourrir la pensée psychanalytique contemporaine, porte à la scène une panoplie de facteurs psychodynamiques, comprenant des éléments préoedipiens, oedipiens et postoedipiens. La présente analyse de l'Oedipe roi met en lumière cette psychodynamique particulière et se distingue sous trois aspects étroitement interreliés. D’abord, l'auteur se centre presque exclusivement sur le texte dramatique plutôt que sur le mythe classique d'Oedipe en lui-même. Dans un deuxième temps, en se basant sur une étude approfondie du texte grec original et de certains signifiants essentiels, l'analyse met en lumière des éléments comme l'étendue de la charge érotique du langage utilisé par Sophocle, jusqu’ici négligée, de même que le caractère suggestif de ses tautologies apparentes quoique conflictuelles. Enfin, l'auteur approfondit tout en l'harmonisant une approche psychanalytique de l'Oedipe roi en utilisant la perspective des spécialistes de la littérature classique qui mettent généralement l'accent sur le rôle des divinités, que minimisent habituellement les psychanalystes. Une intégration herméneutique de l'approche classique de l'arrogance démesurée du héros implique la compréhension du rôle du moi idéal, surtout à la fin de la pièce, à laquelle la littérature classique n'a porté attention que récemment et que la psychanalyse a presque totalement ignorée. Copyright (c) 2010 John Wiley and Sons, ltée.
Ce document est protégé par la loi sur le droit d'auteur. L'utilisation des services d'Érudit (y compris la reproduction) est assujettie à sa politique d'utilisation que vous pouvez consulter en ligne.

https://apropos.erudit.org/fr/usagers/politique-dutilisation/ 


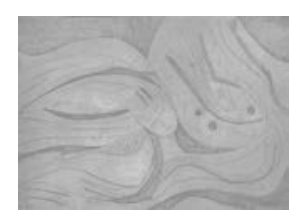

\section{Fdipe roi et la langue de Sophocle ${ }^{1}$}

\section{Patrick Mahony}

Ce classique de Sophocle, qui continue à nourrir la pensée psychanalytique contemporaine, porte à la scène une panoplie de facteurs psychodynamiques, comprenant des éléments préoedipiens, oedipiens et postoedipiens. La présente analyse de I'GFdipe roi met en lumière cette psychodynamique particulière et se distingue sous trois aspects étroitement interreliés. D'abord, l'auteur se centre presque exclusivement sur le texte dramatique plutôt que sur le mythe classique d'Frdipe en lui-même. Dans un deuxième temps, en se basant sur une étude approfondie du texte grec original et de certains signifiants essentiels, l'analyse met en lumière des éléments comme l'étendue de la charge érotique du langage utilisé par Sophocle, jusqu'ici négligée, de même que le caractère suggestif de ses tautologies apparentes quoique conflictuelles. Enfin, I'auteur approfondit tout en I'harmonisant une approche psychanalytique de l'CFdipe roi en utilisant la perspective des spécialistes de la littérature classique qui mettent généralement l'accent sur le rôle des divinités, que minimisent habituellement les psychanalystes. Une intégration herméneutique de l'approche classique de l'arrogance démesurée du héros implique la compréhension du rôle du moi idéal, surtout à la fin de la pièce, à laquelle la littérature classique n'a porté attention que récemment et que la psychanalyse a presque totalement ignorée. Copyright (c) 2010 John Wiley and Sons, Itée.

a pérennité des œuvres classiques tient en partie au fait que, bien qu'elles — soient souvent mal traduites, elles recèlent suffisamment de force pour produire un impact qui se maintient à travers les âges. L'Édipe roi, de Sophocle, fait bien sûr partie de ces œuvres. Il s'agit d'une tragédie extraordinaire, à la fois comme document historique et de par la valeur intrinsèque de son texte. Cette pièce demeure un des documents les plus lus et étudiés dans l'histoire; on la considère comme le premier «suspense» de la littérature occidentale (Segal, 2001,3); à la lumière de nos préoccupations modernes et en effectuant de légères modifications au niveau des références technologiques, on pourrait dire qu'elle décrit un des prototypes les plus célèbres du phénomène moderne que nous désignons sous le vocable «rage au 
volant»; c'est une des œuvres artistiques parmi les plus sublimes, mettant en scène un des plus grands personnages de la dramaturgie grecque, de très grande renommée éponyme subséquente. Enfin, l'Edipe roi met en scène une orchestration unique et inégalée d'ironies qui s'interpénètrent au niveau du langage, des personnages, du thème et de l'intrigue.

D'un point de vue psychologique, l'EEdipe roi témoigne de façon éloquente d'une grande créativité sexagénaire (plus exactement, plusieurs affirment que Sophocle produisit ce chef-d'œuvre au même âge qu'avait Freud lorsqu'il écrivit Le moi et le ça en 1923). En outre - on ne le redira jamais suffisamment -, la pièce de Sophocle a joué un rôle important dans la fondation de la psychanalyse. Comme le souligne Freud, la force particulière de ce texte réside dans le fait qu'il met en scène la vie intrapsychique du spectateur ou du lecteur lui-même par un jeu qui consiste à retarder l'action et à faire monter l'excitation, ce qui constitue un processus multiforme similaire au processus analytique lui-même. Enfin, ce classique de Sophocle, qui continue à nourrir la pensée psychanalytique contemporaine, porte à la scène une panoplie de facteurs psychodynamiques qui comprennent des éléments préoedipiens, oedipiens, et postoedipiens.

Dans le but d'approfondir la psychodynamique complexe de l'Epide roi, l'auteur fonde son analyse sur une étude approfondie du texte grec original, incluant ses signifiants les plus importants ${ }^{2}$. Le résultat de ses recherches détaillées contribue à enrichir de façon significative les données lexicales publiées par Charles Segal, spécialiste reconnu de la littérature classique bien au fait de la psychanalyse. L'examen rigoureux du lexique et des autres complexités discursives du texte grec ont permis à l'auteur de mettre en lumière des preuves additionnelles des motifs et des conflits qui animent les protagonistes de la pièce. La preuve souvent subtile de ces conflits réside dans une polysémie très importante, dans le caractère suggestif des tautologies apparentes et dans la prétendue trivialité de l'ordre dans lequel sont présentées les épithètes. Enfin, l'auteur approfondit tout en l'harmonisant une approche psychanalytique de l'Edipe roi en utilisant l'approche des spécialistes de la littérature classique qui mettent généralement l'emphase sur le rôle des divinités, que minimisent habituellement les psychanalystes. Une intégration herméneutique de l'approche classique de l'arrogance démesurée d'Edipe, qu'intensifie une intelligence hors du commun (Edmunds, 2006, p. 49), implique la compréhension du rôle du moi idéal, surtout à la fin de la pièce, à laquelle la littérature classique n'a porté attention que récemment et que la psychanalyse a presque totalement ignorée. 
Deux précisions méthodologiques s'imposent. D’abord, le lecteur doit prendre en considération l'hésitation habituelle de la psychanalyse à considérer des personnages fictifs comme de vraies personnes. C'est une chose, en effet, de remplir la vie des protagonistes fictifs et celle de leur famille avec de supposés événements absents du texte et ainsi, de spéculer sur ce qui aurait pu ou dû se produire. Mais c'est une tout autre chose de s'inspirer de l'usage que faisait Freud (1921) de la littérature comme preuve textuelle dans la démonstration du conflit inconscient qui se manifeste sous différentes formes de parapraxies.

Deuxièmement, l'approche de l'auteur se distingue de celles de critiques tels Ross $(1982,1995)$ et Priel (2002), qui ont effectué des analyses psychanalytiques approfondies de l'Edipe roi à partir de son contexte mythique. Mentionnons à ce sujet l'attention de plus en plus soutenue portée à la grande indépendance créatrice de Sophocle (Bettini et Guidorizzi, 2004 ; Edmunds, 2006), la description de la nébulosité évocatrice et des modulations complexes, à travers son œuvre, de sa conception tragique du mythe (Garvie, 2005) et enfin, l'excellente recherche de Steiner (1994) sur le contexte culturel des interprétations freudiennes et post freudiennes de la pièce. Mise à part une exception que je m'empresse de justifier ci-après, mon étude porte donc exclusivement sur ce qui est dit dans la pièce en elle-même.

Cette exception concerne le contenu de l'énigme que personne, sauf Edipe, n'arrivait à résoudre. Au sphinx qui demande: "Quel est l'être qui marche à quatre pattes le matin, à deux pattes le midi et à trois pattes le soir?», Edipe donne la bonne réponse: «L'homme». Dans son texte, Sophocle n'énonce pas clairement les termes exacts de l'énigme, pourtant familiers à ses contemporains. Dans une approche typique de sa technique poétique, il fait de nombreuses allusions à l'énigme et à son lien au nom même d'CEdipe (pied enflé) ${ }^{3}$. Par exemple, en indiquant ce qui a causé le délai de son enquête du régicide, CEdipe emploie le mot empodōn (1. 128), qui signifie littéralement «au pied de» et qui est malheureusement souvent traduit par le mot obstacle, et non par le mot latin impedimentum. Sophocle met également dans la bouche de Créon cette réponse suggestive voulant que Thèbes ne puisse faire enquête sur ce mystérieux régicide en raison des problèmes causés par le sphinx qui se trouve «à nos pieds» (pros posi, 1. 131).

À des fins heuristiques, j'aborde l'étude de l'Edipe roi en deux étapes. La première étape porte exclusivement sur les thèmes narcissiques, omniprésents dans la pièce. Dans ce contexte, j'utilise abondamment une clarification fort utile et pénétrante de la conception qu'avait Freud du moi idéal, qui 
jette un nouvel éclairage sur ce grand classique de l'œuvre de Sophocle. Dans la deuxième partie de mon analyse, j'explore la pièce et une partie du même contenu sous une perspective différente. J'y aborde l'intrigue strictement sous l'angle de la théorie de la pulsion et des mécanismes de défense en me concentrant sur une série de scènes dont le contenu oedipien a été jusqu'ici totalement ignoré.

\section{Le nom dans toute sa gloire}

Dans un texte fréquemment cité, Vernant (Vernant et Vidal-Naquet, 1972), éminent helléniste français, critique durement Freud et Anzieu (1966) lorsqu'ils affirment avoir des preuves solides de la présence du complexe d'Edipe dans le chef d'œuvre de Sophocle. Dans un article qu'il intitule de façon provocatrice : "Oedipus without a complex (CEdipe sans complexe)», Vernant les contredit énergiquement tous les deux. Selon lui, la pièce incarne le caractère tragique du destin, qui oppose l'omnipotence divine à la faible volonté de l'humain. Vernant poursuit ainsi: Le propre de la tragédie est de se situer à la frontière de l'interaction entre les actes humains et les pouvoirs divins, là où se révèle leur véritable sens, qui échappe à ceux mêmes qui ont perpétré ces actes et en ont assumé la responsabilité et qui s'insère dans un ordre des choses qui les dépasse et auquel ils n’ont pas accès. Toute tragédie se joue nécessairement à ces deux niveaux. L'interprétation que fait Vernont de la scène du fameux banquet qui a incité CEdipe à consulter l'oracle de Delphes constitue un bon exemple de son approche. Le spécialiste français en vient à la conclusion (p. 92) que cet épisode est nécessaire à la fois au plan esthétique et au plan religieux, mais qu'il n'a rien à voir avec la «psychologie des profondeurs » (appellation qu'il utilise également à la page 94 pour désigner la psychanalyse).

Sans nous laisser influencer par le scepticisme de Vernant à l'égard de la psychanalyse, gardons à l'esprit le thème central de la tension entre le divin et l'humain qu'il propose, comme le font d'autres hellénistes reconnus, comme Knox (1998, pages 47 et 160). Ce dernier émet l'hypothèse voulant que l'Edipe roi, en vertu de la comparaison couramment faite dans la Grèce classique entre le pouvoir des déités et celui des tyrans, réaffirme « une vision religieuse de l'ordonnancement divin de l'univers, une vision fondée sur le concept de l'omniscience divine, représentée dans la pièce par la prophétie d'Apollon. » En effet, les Grecs considéraient que le fait de ne pas faire la différence entre le pouvoir divin et les limites de l'homme pouvait mener à un désastre effroyable. Ainsi, faisant écho à un autre célèbre conseil de l'oracle de 
Delphes prônant la modération (mēdan agan), le chœur de l'Édipe roi critique l'excès (II. 873-878, 1195-1197), qui est une violation de l'idéal grec de modération et du contrôle de soi (sōphrosunē). Par ailleurs, dans son Interprétation des rêves (1900), Freud minimise l'importance de ce motif dans la pièce. Il présume que le thème de la divinité, qui survient relativement tard dans le mythe oedipien, n'est qu'un élément secondaire reconnu comme tel par Sophocle. Un peu plus tard dans son œuvre Introduction à la psychanalyse (1916-1917), Freud précise que l'oracle lui-même servait de réceptacle des désirs oedipiens du protagoniste.

Notre défi initial consiste à harmoniser la signification religieuse de l'oracle pour le public athénien de l'époque avec notre compréhension psychanalytique du narcissisme inhérent au complexe d'CEdipe, à la base de son autoglorification et de sa croyance en son omniscience. Le thème dramatique de la divinité et le rôle du moi idéal, qui correspond au niveau le plus primaire du moi et qui est l'héritier du narcissisme originel, sont le point de départ de notre analyse. Nous verrons que cette profonde dynamique narcissique a eu une influence sur les niveaux ultérieurs de développement de l'estime de luimême d'CEdipe. Dans ce contexte, La gloire et la faute (2006) d'André Lussier est un guide indispensable. En clarifiant la confusion que fait souvent Freud entre le moi idéal et l'idéal du moi, Lussier propose une importante réorientation du complexe d'CEdipe et de ses liens dynamiques avec la pathologie narcissique. Il appert ainsi que les forces déterminantes du narcissisme doivent être considérées comme étant présentes avant, pendant et après le stade oedipien. Les fantaisies narcissiques d'omnipotence, d'omniscience et de perfection du moi idéal sont donc investies de pulsions, car sinon, il n'y aurait que des élaborations de fantaisies inoffensives. L'évolution peut avoir une influence sur l'idéal du moi, mais pas sur le moi idéal, qui est le niveau le plus primitif du moi. Lussier décrit également un conflit multidimensionnel fondamental dans la vie psychique entre les interdits du surmoi et la quête d'une perfection de plus en plus grande, qui provient du moi idéal. Par conséquent, le surmoi n'a d'autre choix que de réagir à l'influence excessive et pathologique du moi idéal.

Venons-en à la pièce elle-même. Un puissant sphinx dominait Thèbes et tuait tous ceux qui n'arrivaient pas à résoudre son énigme. Edipe résout l'énigme, entraînant ainsi la défaite du sphinx, ce qui contribuera plus tard à nourrir sa prétention d'être un surhomme. Imbu du caractère salvateur de sa compréhension, Edipe ne se rendait pas compte de son ignorance des choses terrestres. Il avait une bonne connaissance du développement humain en 
général de l'enfance à l'âge adulte, mais il ignorait son histoire personnelle. En outre, ses connaissances générales plutôt que spéficiques étaient impuissantes à le sauver au plan psychique, car elles n'avaient rien à voir avec une connaissance interdite, réprimée. Ainsi, même après que l'oracle l'ait mis en garde contre son avenir filial, Edipe n'entreprend pas moins d'entrer en relation intense avec deux personnes suffisamment âgées pour être ses parents. Comme le suggère le prophète Tiresias (1l. 440-442), sa perspicacité exceptionnelle s'avéra même contre-productive en ce sens qu' elle contribua à amplifier son orgueil qui, à son tour, accrut sa résistance à découvrir sa vérité intérieure et à reconnaître ses limites par rapport aux dieux. En bref, les changements au niveau de sa soumission révérencieuse aux dieux furent concomitants avec sa confiance grandissante en lui-même, en ses connaissances et en son pouvoir.

Attardons-nous maintenant de plus près au développement du narcissisme d'CEdipe, non pas en suivant les circonvolutions de l'intrigue de la pièce, mais en en reconstituant la séquence historique. Même s'il naquit de Laïus et Jocaste, le couple royal de Thèbes, Edipe fut immédiatement rejeté en raison d'une prophétie menaçante. On perça et on enchaîna les pieds du nouveau-né princier, et on l'envoya mourir dans une montagne lointaine. Une fois arrivé à la montagne, grâce à deux bergers qui devinrent véritablement ses parents sauveurs adoptifs, on lui donna le nom d'Edipe, dont la signification réfère à ses pieds enflés ${ }^{4}$. Je suggère que la fixation ultérieure d'Edipe sur les pieds peut s'expliquer par l'humiliation qu'évoque le verbe grec courant andrapodizō qui signifie : réduire à l'esclavage ou littéralement, enserrer les pieds d'un homme, podes, dans des fers.

L'enfant fut par la suite remis à d'autres parents adoptifs: Polybe et Mérope, roi et reine de Corinthe. Plusieurs années plus tard, un incident fatidique lors d'un banquet éveille les doutes d'Edipe sur ses origines et son statut. La première scène de la pièce explore la rétrospective tardive que fait Oedipe de cet incident de même que les doutes qui changèrent le cours de sa vie (je souligne ici deux allusions cachées aux pieds dans son récit):

J'ai été élevé (à Corinthe) comme le plus grand des citoyens, jusqu'à ce que survienne un événement qui m’étonna, mais sans plus. Au cours d'un dîner, un homme ivre proclama que je n'étais pas le fils de mon père. J'étais outré, si bien que je peinai à me contenir tout au long de la journée. Le lendemain, je me rendis auprès de mon père et de ma mère pour leur poser des questions. Ils infligèrent une punition sévère à l'homme qui avait proféré cette 
insulte. Ils eurent l'impression de m'avoir réconforté, mais cet incident continua à me préoccuper et à me revenir constamment à l'esprit. Je me rendis à Pytho à l'insu de mes parents. (II. 775-788)

Considéré comme le premier des citoyens, le prince est outragé par les propos d'un invité en état d'ébriété avancée. Plus précisément, Edipe fait une description polysémique de cet homme, en utilisant une épithète tautologique, en affirmant qu'il était «trop plein (huperplēstheis) de vin» (à mon avis, le préfixe huper, dont il n'est question que dans une seule des traductions que j'ai consultées (Bowlby, 2007, p. 171), est le plus important des préfixes de toute la pièce). La description d'CEdipe suggère donc que cet invité ivre se tenait difficilement sur ses deux pieds. De plus, Edipe, dans son souvenir, attribue un mouvement à la charge elle-même. Fidèle à son nom de par sa fixation sur les pieds, il est obsédé par cette allusion à sa naissance illégitime : «Cette idée me revenait constamment à l'esprit (hupheirpe)». Il est intéressant de noter que la racine du mot hupheirpe est herpo, qui signifie aussi ramper ou se traîner ${ }^{5}$. En résumé, Edipe, rongé par l'anxiété, est obsédé par cette charge qui l'envahit. Nous pouvons ainsi voir à quel point cette humiliation narcissique l'ébranle profondément. Il accorde plus de crédit à une simple remarque d'un homme en état d'ébriété qu'aux explications ultérieures de ses parents aimants, qui affirment la légitimité de sa naissance. (II. 969-970, 998-999, 1023). Méfiant, CEdipe les quitte, et ce, comme le démontre la suite des événements, pour toujours.

D'une extrême sensibilité narcissique à propos de sa naissance, CEdipe cherche à découvrir la vérité en se rendant à Delphes. Là-bas, l'oracle ignore les questions d'Edipe sur ses origines parentales, ce qui lui inflige une autre blessure narcissique. Il se sent « déshonoré» (atimon, I. 789). Plutôt que de répondre à ses questions, l'oracle lui prédit qu'il tuera son père et couchera avec sa mère, ce qu'Edipe, se considérant comme possiblement adopté, interprète mal comme une confirmation non seulement des allégations de l'ivrogne, mais du mensonge de ses parents bien-aimés à son égard.

Près de Delphes, à la célèbre jonction de trois routes et après qu'il ait entendu la prédiction à l'effet qu'il tuerait son père - donc un homme plus âgé que lui d'une génération - il tue effectivement un homme qui de plus, lui ressemblait physiquement (II. 742-743). Par la suite, comme nous le savons, Edipe se rend à Thèbes, que menace un sphinx sans merci. Selon moi, EEdipe n'était pas conscient qu'il pouvait résoudre la fameuse énigme du sphinx, non seulement parce qu'il était supposément le plus intelligent des hommes, 
mais aussi à cause d'un facteur d'ordre narcissique: sa sensibilité de toujours quant à son image corporelle et à ses pieds blessés le rend plus apte à résoudre l'énigme. Nietzche avait raison d'affirmer, dans Die Geburt der Tragödie (1872/1946), que la réussite d'Edipe n'était pas due à une «sagesse excessive» (seiner übermässigen Weisheit halber).

Aveuglé par sa victoire narcissique de sauveur de Thèbes et par son mariage avec la reine, Edipe fait preuve d'une confiance exagérée en luimême en défiant la menace que représente Thèbes sous la forme des présages de l'oracle suite aux crimes oedipiens. Suppliant CEdipe de sauver la ville une nouvelle fois, un prêtre se complait en éloges contradictoires: il affirme d'abord que le puissant CEdipe a été aidé par un dieu (II. 38-39), puis qu'il l'a été soit par un dieu, soit par un homme (II. 42-43). Enfin, il conclut qu'EEdipe et Apollon sont les deux sauveurs de Thèbes (II. 47-48, 149-150). La fin de la supplique du prêtre suggère même que lui et ses disciples sont à l'autel d'Edipe (II. 15-16). En fait, les flatteries du prêtre indiquent qu'il savait que, sous des dehors de modestie, Edipe avait une perception grandiose de luimême.

Ce n'est que plus tard, alors qu'il est confronté personnellement par le prophète divin Tiresias sur son comportement honteux (I. 367), qu'Edipe abandonne la piété dont il se targuait jusque-là. Incertain quant à son auto glorification, mais tout de même arrogant, il se réclame du rôle de prophète puisque lui seul, et non Tiresias, a réussi à résoudre l'énigme du sphinx. De plus, il prétend exercer son don de prophétie sans l'aide des dieux - ce qui contredit la déclaration qu'il avait faite précédemment selon laquelle il vaincrait avec l'aide des dieux ou qu'il périrait (II. 145-146). À ce point, EEdipe considère qu'il ne peut être qu'un objet d'envie - plutôt que d'être sujet à l'envie - comme il l'affirme en exultant: "Ô richesse, royauté et indicible adresse dans une vie enviée de tous» (II. 380-381, emphase ajoutée par l'auteur).

L'apparition de Jocaste marque un autre tournant dans la teneur religieuse de la pièce. Cette présence offre un cadre à l'intérieur duquel nous pouvons suivre les hésitations d'Edipe entre l'auto idéalisation et la peur de l'auto dépréciation. À mesure qu'il apprend les détails du régicide, il passe d'une position à l'autre: «Ô Zeus, qu'avez-vous fait de moi ?... J'ai de forts doutes quant à la clairvoyance du prophète... Ne serait-il pas juste de dire que mon destin provient de l'action d'une déité cruelle?» (II. 738, 747, 828829).

Confus, le fier CEdipe se voit dans l'obligation de faire enquête sur ses origines auprès d'un messager corinthien, un travailleur immigrant (thèteai, 
I. 1029) qui faisait partie de la catégorie des hommes libres dont le statut surpassait de peu celui d'esclave. Les vieilles craintes d'Edipe sur ses origines sont rapidement ravivées lorsqu'il réalise que Polybe n'est pas son père. Apprenant également qu'il a été sauvé par un berger corinthien, Edipe poursuit: "De quel trouble étais-je affligé lorsque tu me pris dans tes bras?» (I. 1031). Quand le messager lui dit que ses chevilles avaient été percées, le roi se plaint d'avoir à se rappeler ses souffrances d'antan, puisque ses pieds percés permettent de l'identifier et sont même à l'origine de son nom. Il n'y a rien d'étonnant au fait que la prise de conscience aigüe que fait CEdipe de son histoire physique et de sa naissance supposément de bas étage accroît sa blessure narcissique et l'amène à ressentir la honte qui l'habite depuis toujours. Le choix surdéterminé des mots qu'il utilise dans sa lamentation revêt une signification autobiographique cachée: «Une honte terrible de mes langes s'empara de moi » (I. 1035). Plus exactement, le mot grec pour «s'emparer de » (aneilomēn) fait référence à ce qui suit:

(1) Une coutume qui avait cours alors selon laquelle on attachait autour du cou des enfants de petits objets qui permettraient plus tard de les reconnaître (comme le fit Créuse, pleine d'espoir, dans le Ion d'Euripide). Il est possible aussi que par cette remarque, Edipe indique qu'il est affligé depuis la naissance d'une condition déshonorante (CEdipe roi, sous la dir. de Jebb et Easterling, 2004, p. 138n).

(2) dans le terme "s'emparer de», on fait ironiquement allusion à la coutume grecque qui voulait qu'un père "s'empare» de son enfant, reconnaissant ainsi le nourrisson comme son fils légitime (EEdipe roi, sous la dir. de Blondell, 2002, p. 67n).

Bouleversé par son statut social d'enfant illégitime et d'objet de mutilation, considérée alors comme une tare distinguant les esclaves des nobles dans la Grèce classique, Edipe, d'un ton moqueur, évoque devant Jocaste la possibilité qu'il ne soit pas un esclave récent, mais un esclave issu d'une lignée générationnelle: "Ne t'inquiète pas! Même s'il s'avère que je sois le descendant de troisième génération d'une lignée d'esclaves, tu ne seras jamais de basse naissance!» (II. 1062-1063). Puis, il affirme rapidement qu'à l'inverse, Jocaste est fière de ses propres origines nobles (II. 1069-1070). Éperdue et désillusionnée, Jocaste se retire dans le but de se suicider. Laissé à lui-même, Edipe s'imagine que, comme toute femme fière, Jocaste a honte de ses origines modestes (I. 1079). Puis, dans un effort désespéré pour surmonter sa blessure narcissique, Edipe, grandiloquent, affirme avoir vaincu les vicissitudes de la naissance et de la croissance humaines. Il prétend qu'il est le fils 
de Chance, qui domine et personnifie le manque d'ordre cosmique de l'univers (II. 1080-1083), devenant ainsi «l'égal des dieux, le fils de Chance, la seule vraie déesse» (Knox, 1998, 156fn).

André Green (1992,110n) affirme qu'Oedipe surmonte sa déception de n'être pas fils de roi - une condition qu'il attribue par projection à Jocaste en se réclamant de parents divins, ce qui constitue une projection de son moi idéal. On pourrait aussi dire que la force de son moi idéal est telle qu'elle transcende toute condamnation du surmoi. Un renversement majeur survient par la victoire absolue du surmoi lorsqu'Oedipe, ayant perdu ses illusions, se crève les yeux, ce qui le punit jusqu'à la fin de ses jours en Hadès.

Pour approfondir notre compréhension de la pièce, il est essentiel de souligner qu'après avoir prétendu être né de Chance, Edipe ne fait aucunement référence à la divinité dans les deux cents lignes suivantes au cours desquelles est atteint un paroxysme dramatique. Son propos se situe de plus en plus au niveau humain, dans sa recherche de ses origines et dans son autopunition. Mis à part le bref commentaire de Michels (1986) qui constitue une exception de taille, les analyses se sont centrées sur le paroxysme dramatique de la pièce en négligeant sa conclusion, occultant ainsi son importance dans le changement d'équilibre chez Edipe entre les forces du moi idéal et celles du surmoi.

Retraçons maintenant les étapes du revirement qui amène CEdipe à se crever les yeux. À un niveau supérieur, il reconnaît son asservissement aux dieux, mais au niveau humain, il exprime une appréciation triomphante de ses actes maudits due à la puissance même de ces actes. Ainsi, après s'être lamenté sur son impuissance, il se ressaisit suffisamment pour pouvoir glorifier la puissance funeste de son ignominie: impardonnables, ses crimes filiaux sont "plus puissants» (kreisson', I. 1374) que toute tentative d'expiation qu'il aurait pu faire en se pendant. Ensuite, faisant fi de son vif sentiment d'humiliation, il affirme que lui et sa mère étaient exempts de différenciation en tant qu'êtres humains, sans rôles précis et distincts dans la structure familiale: «Mariage, mariage, vous m’avez donné la vie, puis fait grandir ce même germe, et exhibé des pères qui étaient frères, des enfants qui étaient le fruit de l'inceste, des épouses qui étaient à la fois épouses et mères du même homme» (II.1403-1407; cf. Pucci, 1992). Remarquons que c'est à ce moment que la trivialité réductrice de l'identité d'Edipe s'éloigne le plus de la singularité des impératifs de son moi idéal.

Après s'être de nouveau ressaisi, Edipe réagit en affirmant avec grandiloquence sa singularité, en se situant hors du contexte familial. Arrivant à un 
compromis en créant un nouvel équilibre entre les pressions de son surmoi et celles de son moi idéal, Oedipe, roi incontesté de l'adversité, proclame: «Aucun autre être humain ne pourrait porter le fardeau qui est le mien» (II. 1414-1415). S'étant ainsi réaffirmé avec autant d'ambivalence, Edipe cesse d'être habité par la peur comme il l'était auparavant. Il va même jusqu'à dire au chœur d'arrêter d'avoir peur, même s'il désigne Créon comme «le meilleur des hommes » et se décrit lui-même comme «le pire des êtres humains » (I. 1443).

Les derniers échanges entre CEdipe et Créon sont empreints d'ambivalence. D'une part, Edipe exprime son obédience envers Créon et d'autre part, il lui intime l'ordre d'assurer le bien-être de ses filles. Créon manifeste sa contrariété en répliquant: «Ne cherche pas à exercer ton pouvoir sur toute chose! Ce pouvoir ne t'a pas été accordé tout au long de ta vie» (II. 15231524). Cette sommation de Créon illustre le contraste qui apparaît à la fin de la vie d'CEdipe entre son attitude envers les dieux et envers les hommes. D'un côté, nous devons conclure que la fin de la pièce donne raison aux oracles, à la connaissance divine et à leurs conséquences: même si les dieux peuvent se montrer injustes, ils savent (Burkert, 1991). En même temps, nous voyons que les prétentions du moi idéal d'CEdipe ne furent que partiellement jugulées: dans sa grandiloquence obstinée, il se sentait unique dans l'adversité.

\section{Un nouveau commencement: le retour du refoulé}

Nous n'aurons de cesse de poursuivre notre exploration Au bout de tous nos efforts,

Nous arriverons là d'où nous sommes partis et connaîtrons vraiment cet endroit pour la première fois. T.S. Eliot, Les quatre quartets: «Little Gidding»

En résumé: dans son effort pour trouver le régicide responsable de la peste sévissant sur Thèbes, Edipe s'entretient avec Créon. Au cours de cette conversation, il fait tour à tour allusion à un seul tueur et à un groupe de meurtriers (II. 124-241).

Edipe rencontre ensuite Tiresias, le devin aveugle. Leur discussion s'étend aux thèmes oedipiens du parricide et de l'inceste. Tiresias lance alors cette accusation: "J'affirme que vous êtes le meurtrier de l'homme dont vous cherchez le meurtrier» (I. 363). Tiresias explique alors la malédiction dont Edipe a hérité de ses parents et souligne le côté scandaleux de son union matrimoniale. Plutôt que d'admettre que la déclaration de Tiresias fait écho aux prédictions d'Apollon, Oedipe réagit avec effronterie et se répand en injures sur la folie de Tiresias. À ce point, Tiresias réplique que même les 
parents qui ont "donné naissance » à Edipe reconnaissaient la sagesse du devin (I. 436). La tautologie apparente de l'expression «les parents qui donnèrent naissance » éveille à nouveau les doutes d'CEdipe quant à ses origines et le plonge dans la confusion. Désarmé par le retour à la surface de ses propres refoulements, celui qui avait résolu l'énigme du sphinx ne peut que conclure au caractère énigmatique des propos de Tiresias.

Lors de la rencontre subséquente entre CEdipe et Jocaste, la question numérique atteint son apogée. Ayant fait allusion à un meurtrier lors de sa discussion préalable avec Créon et à deux parents avec Tirésias, Edipe porte alors son attention sur les trois routes à l'intersection desquelles a eu lieu le meurtre de Laïus (Edipe roi, sous la dir. de Dawe, 2006, p. 3). Ce soi-disant détail, que Jocaste mentionne rapidement dans son récit exhaustif des événements, précipite Edipe dans une confession de désarroi psychique: «Femme, tes propos suscitent chez moi un grand questionnement et bouleversent mon esprit!» (II. 726-727). Craignant de ne pas avoir bien entendu, Edipe exprime une hésitation qui ne se rencontre nulle part ailleurs dans la pièce: «Je crois vous avoir entendu dire que Laïus a été assassiné là où se rencontrent les trois routes» (II. 729-730). Les précisions qu'ajoute Jocaste sur la localisation de ces trois routes sont érotiquement très suggestives: «C'est dans le pays de Phocis que la route se divise: une de ses branches part de Delphes et la troisième de Daulis » (II. 733-734) ${ }^{6}$.

Effrayé par ces propos, Edipe entreprend une reconstruction défensive du régicide. Il commence par faire une distorsion chronologique des événements afin de se distancier du mort. Ainsi, présumant de l'âge de Laïus au moment de sa mort, CEdipe, lui-même alors d'âge moyen, s'enquiert : "Quel stade de sa jeunesse (akmēn hēbès) avait-il atteint?» (I. 741). En répliquant ainsi, Oedipe démontre aussi qu'il s'identifie à Laïus. En effet, parmi tous les termes qu'il aurait pu choisir pour exprimer l'idée d' «atteindre», Edipe, de par sa fixation sur les pieds, utilise à nouveau le mot heirpe qui, comme nous l'avons vu dans la scène du banquet, signifie aussi « ramper». Comme s'il utilisait une autre référence inconsciente à son enfance, Edipe, dans son identification à son père, exprime l'idée que lui et son père étaient dans le processus de «ramper» vers la maturité.

Mais il y a plus. Lorsqu'CEdipe apprend que contrairement à son hypothèse désespérée, le roi était d'âge moyen au moment de sa mort (II. 742743), il tente à nouveau de prendre ses désirs pour la réalité en posant une question sur le nombre de gardes qui accompagnaient le roi: «Se déplaçaitil alors avec une petite escorte ou, à la manière d'un roi, était-il accompagné 
par de nombreux gardes?» (II. 750-751). Rejetant les extrêmes auxquels Edipe fait référence dans son désir d'éviter la réalité, Jocaste mentionne que le nombre de personnes qui accompagnaient Laïus n'était ni élevé ni faible, mais quelque part entre les deux : «Il y avait cinq personnes en tout dont un héraut, en plus du char qui transportait Laïus» (II. 752-753). Jocaste apporte une distinction importante sur l'ampleur de l'escorte par le terme qu'elle utilise pour désigner le char de Laïus.

Un $a p \bar{e} n \bar{e}$ était en fait un véhicule à quatre roues. Mené par un héraut à la tête d'une escorte, il s'agissait d'un convoi royal (CEdipe roi, sous la dir. de Jebb et Eastering, 2004, p. 104 ; sous la dir. de Blondell, 2002, p. 54). La pression de la réalité se refermant peu à peu sur lui, les distorsions d'CEdipe atteignent leur apogée quand il fait à nouveau allusion à l'âge de Laïus. Après qu'on lui ait révélé que le roi n'était pas dans sa jeunesse, mais au mitan de sa vie, Oedipe, lui-même d'âge moyen, se distancie à nouveau temporellement du roi en y référant comme à un «vieil homme» (presbus, II. 805, 807) ${ }^{7}$ qui avait tenté de l'écarter de son chemin.

D'autres dérives verbales illustrent la relation conflictuelle entre CEdipe et sa mère. Dans un effort pour détourner l'attention d'Oedipe de l'histoire de ses pieds percés, Jocaste décrit le sort de son fils unique soi-disant mort et dont les pieds n'avaient été qu'attachés (enzeuxas, I. 718). Le choix du mot qu'utilise Jocaste - zeugnumi, qui peut aussi vouloir dire « unis par les liens du mariage » — révèle-t-il un déplacement et un retour à la surface du refoulement? Peu après, Edipe répète la prévision de l'oracle lorsqu'il se pose tout haut la question suivante, formulée d'une façon particulière en évoquant la fonction paternelle: "Serai-je uni (zugēnai) par les liens du mariage avec ma mère et tuerai-je mon père Polybus, celui-là même qui m’a élevé et engendré? (II. 825-827). Par cette question qui illustre son angoisse surdéterminée, Edipe inverse involontairement la séquence chronologique de la fonction paternelle, révélant ainsi la réalité primaire de son père adoptif Polybus en tant que père nourricier, de même que sa fantaisie ultérieure de lui en tant que géniteur. Devant le désarroi de son mari face à ce qu'elle lui a révélé, Jocaste lui demande: "Que t'ai-je dit qui te semble particulier? (I. 841). Par ailleurs, le mot grec pour "particulier» (perisson) peut aussi signifier « excessif», ce qui met encore davantage en relief la culpabilité croissante qu'induisent chez Edipe les propos de sa mère.

Lorsque le messager arrive de Corinthe pour annoncer la mort de son supposé père, Oedipe se croit partiellement au-delà des prédictions de l'oracle de Delphes. Même s'il écarte la possibilité du parricide, il craint encore 
l'inceste avec sa supposée mère Mérope. Il est particulièrement intéressant de noter la façon dont il désigne Mérope, non pas en fonction de son statut civil, mais en termes de cohabitation: "Celle qui vivait auprès de Polybe » (I. 990). Ce détour linguistique, malencontreusement qualifié d'inutile (Edipe roi, sous la dir. de Dawe, 2006, p. 159), prend en fait tout son sens si nous considérons à quel point il reflète l'angoisse croissante d'CEdipe quant à sa propre cohabitation interdite avec Jocaste.

Une analyse contrastive approfondie des échanges ultérieurs entre Jocaste et CEdipe démontre davantage le caractère subtilement défensif de leurs choix lexicaux. Fait révélateur d'une autocensure érotiquement chargée, Jocaste n'utilise jamais d'épithète relative à la procréation pour remplacer ou faire référence élaborée à la «mère » ou au «père», même dans des phrases comme «le parent qui a donné naissance à un enfant». Pourtant, de telles épithètes étaient fréquemment utilisées dans le grec ancien. De plus, elle n'utilise jamais les mots «père» et «mère» dans la même phrase. À l'opposé, CEdipe réfère volontiers à son père et à sa mère simultanément soit en les désignant directement, soit en faisant référence à leur potentiel procréateur, par exemple dans le passage suivant: "Je n'irai jamais là où se trouvent ceux qui m'ont procréé» (I. 1007) ${ }^{8}$.

Edipe ne peut faire référence à l'identité paternelle que lorsqu'il ne parle que de son père. Selon Edipe, l'oracle avait prédit qu'il mentirait à sa mère et tuerait «le père qui l'avait engendré» (II. 791-793). Par ailleurs, Edipe censure ses propos lorsqu'il parle à Jocaste de la maternité. Il peut parler de la mère en elle-même avec parcimonie, mais il n'utilise aucun terme additionnel pour qualifier son identité maternelle. Il est intéressant de noter que ce n'est qu'au moment de la mort de Jocaste qu'CEdipe fait allusion à son identité maternelle, ayant même recours à une périphrase pour remplacer le mot mère: «Pauvre créature, était-ce réellement celle même qui enfanta?» (I. 1175). Adoptant une attitude de détachement défensif, Oedipe ne fait jamais référence à la dépouille de Jocaste lorsqu'il somme Créon de faire son devoir et d'enterrer «celle qui se trouve dans la maison... Celle qui est la vôtre» (II. 1447-1448). Ce nouveau refoulement s'étend non seulement aux simples références lexicales que fait Oedipe à ses fautes oedipennes, mais aussi à leur ordre syntaxique. Dès lors, avant la mort de Jocaste, il mentionne toujours d'abord l'inceste, puis le parricide - comme en une réaction mimétique à une réalité vivante (II. 791-793, 825-827, 994-996, 1184-1185). Cependant, après la mort de Jocaste, il renverse cet ordre chronologique en mentionnant en premier le parricide (II. 1357 - 1359, 1398 - 1408). 
Au moment où il prend finalement conscience de la situation, Edipe réclame un sabre et se précipite dans la chambre de Jocaste pour la trouver pendue. Frustré dans son désir probable de matricide, Edipe se crève les yeux. Une série de questions surgissent alors: de quelle nature était cet acte par lequel CEdipe s'est enlevé la vue? Pourquoi CEdipe n'a-t-il pas plutôt choisi de s'arracher les yeux, ce qui l'aurait aussi rendu aveugle? Pourquoi at-il utilisé un instrument qu'il a retiré du corps de Jocaste pour se crever les yeux? Pour trouver réponse à ces questions, retournons une fois de plus au texte. Sophocle fait décrire cette scène violente par un messager en utilisant des termes empreints de significations oedipennes: «Il arracha de sa robe des épingles d'or qui l'ornaient puis, levant les yeux au ciel, les creva» (II. 1267-1269). À mon avis, les épingles qu’il utilisa pour se crever les yeux sont surdéterminées, car le terme utilisé en grec pour les désigner peut aussi signifier les os externes de l'avant-bras et de la jambe, c'est-à-dire le radius et le péroné. À un niveau plus large, Segal (2001, p. 127) ajoute qu'en retirant les longues épingles qui tenaient ensemble les pans de la robe de Jocaste, c'est un peu comme si Edipe la déshabillait dans leur chambre conjugale. En outre, dans la littérature grecque classique, les yeux étaient souvent désignés comme étant le siège de l'érotisme par lequel la lumière surgit et influence l'autre (voir Antigone, I. 795). Ainsi, en effectuant un déplacement et en substituant symboliquement ses propres orbites aux organes génitaux féminins (Henderson, 1991, p. 130), Edipe s'invagine lui-même.

Je crois également qu'il faut prendre en considération le sens du verbe paīo qui, utilisé à deux reprises par le messager pour décrire le geste d'CEdipe quand celui-ci se crève les yeux, signifie aussi copuler - ce qui souligne la polyvalence du geste posé par Edipe. En enfonçant dans ses yeux cet objet symboliquement érotique provenant des vêtements que porte le corps de son épouse, CEdipe effectue un geste surdéterminé, c'est-à-dire une agression qui masque une performance libidinale de même qu'un coït violent avec luimême partiellement identifié à Jocaste. En même temps, cet acte d'auto castration reproduit le perçage de ses pieds (I. 1034) dont l'enflure peut évoquer une érection (Freud, 1974, p. 163).

Du point de vue du surmoi, il est à noter qu'en voulant réaliser tardivement et de façon masochiste le désir infanticide de ses parents, Edipe se résout à être abandonné pour toujours sur le mont Cithéron (II. 1451-1453; cf. 1391-1392). Cette décision prise par Edipe provient, à mon avis, d'une fantaisie remarquable et jusqu'ici ignorée d'un cercle vicieux qui illustre sa vie de la façon suivante: Edipe commence son cycle de vie à Thèbes; il est 
ensuite amené dans le sud-est au mont Cithéron puis à Corinthe; de là, il se rend au nord-ouest à Delphes, que les Grecs considèrent comme le nombril du monde. Il poursuit alors sa route vers l'est et tue son père dans une route étroite qui symbolise le vagin puis, fermant ce cercle vicieux fatidique, se rend encore plus à l'est pour jouir sexuellement de sa mère à Thèbes.

À voir le cercle vicieux de ces désirs, Edipe se sent fautif à un point tel qu'il se coupe brutalement de toute forme de plaisir, incluant la possibilité de voir ses enfants (II. 1375-1377). On ne mesure la véritable sévérité du surmoi d'CEdipe que lorsqu'on prend en compte la croyance qu'avaient les Grecs que les morts conservent leur intégrité physique ou leurs blessures quand ils vont au royaume de Hadès (II. 1371-1372; voir aussi Edipe roi, sous la dir. de Kamerbeek, 1967, p. 249). Ainsi, étant donné la profonde conviction qu'avait Edipe du caractère impardonnable de sa faute, c'est comme si, non satisfait du fait que son surmoi devenait l'héritier de son complexe d'Edipe pour la durée de son existence, celui-ci s'inflige une punition éternelle. La vie œedipienne a une limite temporelle. La souffrance postoedipienne n'en a pas.

\section{Conclusion}

Du point de vue psychanalytique, Edipe roi se démarque par la description qui y est faite de l'aveuglement et des prises de conscience du héros. Moyennant sa propre souffrance et ses réflexions sur certaines preuves concrètes, Edipe acquiert une information généalogique superficielle pour comprendre la nature incestueuse et meurtrière de ses actes, mais ironiquement, l'incarnation même du complexe d'CEdipe reste dans l'ignorance quant à son propre complexe. Il n'acquiert qu'une connaissance très partielle de sa propre humanité oedipienne, seulement ce qu'avaient prédit les dieux, et sa mauvaise compréhension a amplifié sa prise de conscience différée. Il interprète ses difficultés comme le résultat du désir qu'avaient les dieux de faire de lui l'auteur ignorant d'actes défendus. En résumé, inconscient de ses propres enjeux oedipiens, Edipe était conscient de ses ambitions préoedipiennes, mais non, bien sûr, de leur profondeur archaïque ni de la multiplicité de leurs manifestations. Par ailleurs, sa détermination courageuse à subir son destin vient de sa nature propre et non des dieux (Knox, 1964, p. 27). Il accède ainsi à nouveau à une certaine grandeur, mais d'un autre ordre.

Chez CEdipe, il n'y a rien de "politiquement correct» comme on le trouve chez Créon. Même plongé dans les ténèbres de l'ignorance, il continue avec un courage héroïque en s'abstenant de toute adaptation et en accordant une valeur primordiale à la poursuite de la vérité sans égard aux coûts personnels 
que celle-ci entraîne. Il se fait le champion incontesté d'un idéal diamétralement opposé à celui proposé par Baby Sugg dans Beloved de Toni Morrisson (1987, p. 102) : «Tout dépend de la connaissance qu'on a du moment où il faut s'arrêter. » On pourrait néanmoins affirmer que le moi idéal d'Oedipe était mobilisé par une poursuite de la vérité qu'il menait à la fois avec une grandeur héroïque et avec démesure. La connaissance qu'acquiert finalement Edipe ne l'apaise pas: elle est plutôt tragique. Son destin résume à lui seul le sombre truisme du texte médiéval Der Ackermann aus Böhmen (von Tepl, 1951), voulant que dès sa naissance, l'homme est suffisamment âgé pour mourir. Le destin d'Edipe illustre également l'idée qu'exprime E.M. Forster dans Howard's End (1910) à l'effet que même si la mort nous détruit, l'idée de la mort peut nous sauver. Même si elle a fait de lui un être handicapé et aveugle, l'idée de la mort a sauvé CEdipe.

Je devrais aussi arguer que l'exploitation que fait Sophocle du nom d'CEdipe constitue un matériel très riche pour l'exploration analytique de l'incroyable portée universelle de l'œuvre de ce génie créateur. Edipe roi est un chef-d'œuvre mondial inégalé: les signifiants et les signifiés évoqués dans son titre original et donc dans le nom du personnage principal, Oidipous (le mot tyrannos fut ajouté au quatrième siècle), font non seulement partie intégrante de l'intrigue, mais indiquent en eux-mêmes son sens profond, ce qui est révélateur des résonances inconscientes et préconscientes contenues dans le texte grec original ${ }^{9}$. Constituant le signifiant principal de la pièce, les trois premières lettres du nom du héros réferent (1) au verbe oida, qui signifie « avoir vu» et « connaître»; (2) au verbe oideō, qui signifie « enfler» physiquement ou mentalement (par exemple: les pieds enflés et la grandiloquence du héros). Le mot pous, en plus de désigner le pied, contient le mot pou qui, selon qu'il soit accentué ou non, peut signifier «peut-être» ou «où ». L'atmosphère d'incertitude qui imprègne la pièce est peut-être indicatrice des nombreux indécidables qu'elle recèle, tandis que le mot où récapitule les deux thèmes dramatiquement décisifs de la place et du mouvement (Corinthe, Thèbes, Delphes; les nombreuses routes et les champs, de même que les espaces situés à l'extérieur et à l'intérieur du palais et de ses appartements). Qui plus est, le mouvement en lui-même est à la fois physique et mental, par exemple le vagabondage et même l'errance de la pensée, comme peut signifier le mot grec planō (II. 67, 301, 727, 1029). En outre, les six dernières lettres du nom du protagoniste forment un autre mot - dipous (deux pieds) - dont la signification représente la deuxième étape du développement dans l'énigme du sphinx, c'est-à-dire le passage de l'enfance à la maturité et à la faiblesse de la vieillesse. 
Nous nous devons de conclure que la pièce de Sophocle, qu'il s'agisse du texte original ou de sa traduction, pose un défi aux psychanalystes, qu'ils soient lecteurs ou spectateurs de celle-ci. De par son génie créateur, Sophocle crée chez le lecteur des désirs de résolution, puis frustre souvent ces désirs, que ceux-ci soient narcissiques ou d'une autre nature. Idéalement, une telle absence de résolution demande que nous nous abstenions de conclure la pièce de façon non justifiée et qu'en toute objectivité envers ce chef-d'œuvre, nous maintenions cette capacité négative que Keats réclamait des poètes euxmêmes. Même si EEdipe roi représente certainement «la tragédie paradigmatique de l'aveuglement de l'homme» (Kaufman, 1968, p. 120), le risque demeure toujours que dans une identification excessive et trompeuse, nous soyons tentés de croire que l'héroïsme exceptionnel d'CEdipe soit paradigmatique de notre propre vie. En fait, à cause de l'incroyable complexité et des multiples attraits d'Edipe roi, nous pouvons parfois, en relisant la pièce, faire de nouvelles découvertes et parfois réprimer certains éléments, tout en essayant de garder à l'esprit les différentes réverbérations pré-œdipiennes, oedipiennes et postoedipiennes du nom «Edipe». Même incomplète, une telle conscience ajoute à notre richesse en tant qu'analystes qui étudient Edipe roi à la fois pour s'en inspirer et pour confirmer certains principes psychanalytiques fondamentaux.

\section{Notes}

1. Traduction de Mahony, P. (2010), The Oedipus Rex of Sophocles and Psychoanalysis, International Journal of Applied Psychoanalytic Studies, 7, 290-306.

2. Dans cet article, mes citations proviennent en majorité de la traduction classique d'CEdipe roi par le grand spécialiste de Sophocle, Hugh Lloyd-Jones (Sophocles, 1994a, 1994b). Cependant, certaines traductions sont de moi, lorsque de plus amples précisions littérales sont nécessaires. J'utilise des caractères normaux pour les numéros des lignes lorsque je cite des traductions de Lloyd-Jones alors que j'utilise l'italique lorsque je cite mes propres traductions. Par exemple, le chiffre 128 dans le texte ci-dessous indique qu'il s'agit d'une traduction de Lloyd-Jones alors que 128 indiquerait qu'il s'agit d'une de mes traductions.

3. Mentionnons ici que les analyses d'Edipe roi effectuées par Freud ne réfèrent jamais au texte original, mais à sa traduction en allemand, ce qui est étrange compte tenu du fait qu'il rédigeait son journal quotidien en grec lorsqu'il était jeune. Je soupçonne que ce fait a été ignoré par les chercheurs parce que Jones l'a déplacé pour le relater dans le deuxième tome de sa biographie (1955, p. 24).

4. Il faut dire que certains spécialistes des lettres classiques, comme Dawe, dans son édition d'Edipe roi (2006), mettaient en doute le lien entre l'énigme du sphinx, la blessure d'Edipe et les références aux pieds contenues dans la pièce.

5. Edipe devait son nom aux bergers qui l'avaient secouru et non à ses parents (comme il l'affirme plus tard erronément) (1. 1037). Comme Bollack l'explique (1991, vol. 3), ses parents naturels n'auraient pas nommé l'enfant dont ils voulaient se débarrasser, surtout qu’ils n'auraient pu ni prévoir ni souhaiter que cet enfant aux pieds enflés survive. 
5. Bowlby (2007, p. 171) indique avec raison que le sens littéral du mot hupheirpe est « ramper». Par ailleurs, il ne fait pas de lien avec les références au pied dans la pièce.

6. Dans cette réplique très suggestive, Jocaste utilise l'adjectif prédicatif schistē (diviser), qui est le mot utilisé dans la langue argotique pour désigner une fente ou une crevasse et qui peut également référer au vagin (Henderson, 1991, p. 147).

7. Edipe utilise le mot presbus de façon plus appropriée pour le messager corinthien (I. 1103) et pour le chœur (I. 1111).

8. Lloyd-Jones traduit simplement cette périphrase apparemment inutile par "parents». Dans une pièce dont l'intrigue tourne autant autour de la distinction entre les parents naturels et les parents adoptifs, les qualificatifs parentaux ne sont pas du tout superflu. Dans sa traduction des lignes 1012 1017, 1082 et 1497, Lloyd-Jones réduit une fois de plus des éléments spécifiques cruciaux aux termes "parents », «mère » et «père».

9. La complexité linguistique de l'EEdipe roi devient évidente lorsqu'on la compare à celle de la célèbre légende ovidienne de Narcisse. Malgré sa puissance poétique, l'étude du texte latin d'Ovide ne permet pas d'identifier de signifiants de la pièce dans le nom de Narcisse, même si son interaction avec Écho pourrait, dans une certaine mesure, nous faire attendre le contraire.

\author{
Patrick Mahony, PhD \\ 1297, Saint-Viateur \\ Outremont (Québec) H2V $1 Z 2$ \\ mahony@videotron.ca
}

\title{
Références
}

ANZIEU, D., 1966, CEdipe avant le complexe. Les Temps Modernes, 245, 675-715.

BETTINI, M., GUIDORIZZI, G., 2004, Il mito di Edipo: immagini e racconti dalla Grecia, Einaudi, Torino.

BOWLBY, R., 2007, Freudian Mythologies, Oxford University Press, Oxford.

BURKERT, W., 1991, Oedipus, Oracles, Toronto University Press, Toronto.

ELIOT, T. S., 1963, Collected Poems : 1909-1962, Harbrace, New York.

EDMUNDS, L., 2006, Oedipus, Routledge, New York.

EURIPIDES, 1958, Hecuba, Andromache, The Trojan Women, Ion, D. Grene dir., (R. Willetts trad.), Chicago University Press, Chicago, IL.

FORSTER, E., 1910, Howard's End, Vintage Press, New York.

FREUD, S., 1900, The Interpretation of Dreams, Standard Edition, 4.

FREUD, S., 1901, The Psychopathology of Everyday Life, Standard Edition, 6.

FREUD, S., 1916 - 1917, Introductory Lectures on Psychoanalysis, Standard Edition, 16.

FREUD, S., 1923, The Ego and the Id, Standard Edition, 19, 3-68.

FREUD, S., 1974, The Freud/Jung Letters: 1906-1914, W. McGuire dir., (R. Hull trad.), Princeton University Press, Princeton, NJ.

GARVIE, A., 2005, The Plays of Sophocles, Bristol Classical Press, London.

GREEN, A., 1992, La déliaison, Les Belles Lettres, Paris.

HENDERSON, J., 1991, The Maculate Muse: Obscene Language in Attic Comedy, Oxford University Press, Oxford.

JONES, E., 1955, The Life and Work of Sigmund Freud, vol. 2, Basic Books, New York.

KAUFMANN, W., 1968, Tragedy and Philosophy, Princeton University Press, Princeton, NJ. 
KNOX, B., 1964, The Heroic Temper: Studies in Sophoclean Tragedy, University of California Press, Berkeley, CA.

KNOX, B., 1998, Oedipus at Thebes: Sophocles' Tragic Hero and his Time, $2^{\mathrm{e}}$ éd., Yale University Press, New Haven, CT.

LUSSIER, A., 2006, La gloire et la faute, Presses de l'Université du Québec, Sainte-Foy.

MICHELS, B., 1986, Oedipus and insight, Psychoanalytic Quarterly, 55, 599-617.

MORRISON, T., 1987, Beloved, Vintage, New York.

NIETZSCHE, F., 1946, Die Geburt der Tragödie, A. Kröner, Stuttgart, (éd. originale 1872).

OVID, 1916, Metamorphoses (tome 1), F. Miller dir., William Heinemann, London.

PRIEL, B., 2002, Who killed Oedipus? International Journal of Psychoanalysis, 87, 433-443.

PUCCI, P., 1992, Oedipus and the Fabrication of the Father, Johns Hopkins University Press, Baltimore, MD.

ROSS, J., 1982, Oedipus revisited, Psychoanalytic Study of the Child, 37, 169-200.

ROSS, J., 1995, King Oedipus and the postmodernist psychoanalyst, Journal of the American Psychoanalytic Association, 43, 553-571.

SEGAL, C., 1994, Sophocles' Oedipus tyrannus: Freud, language, and the unconscious, in Rudnytsky, P., and Spitz, E., eds., Freud and the Forbidden Language, New York University Press, 72-95.

SEGAL, C., 1995, Sophocles' Tragic World: Divinity, Nature, Society, Harvard University Press, Cambridge, MA.

SEGAL, C., 1999, Tragedy and Civilization: An Interpretation of Sophocles, Oklahoma University Press, Norman, OK.

SEGAL, C., 2001, Oedipus Tyrannus: Tragic Heroism and the Limits of Knowledge, Oxford University Press, New York.

SOPHOCLES, 1967, The Plays of Sophocles, Vol. 4, The Oedipus Tyrannus, J. Kamerbeek, E.J. Brill, Leiden.

SOPHOCLES, 1991, L'Edipe roi de Sophocles, Bollack, J., éd., Presses universitaires de Lille.

SOPHOCLES, 1994a, Ajax, Electra, Oedipus Tyrannus, Lloyd-Jones, H., éd., Harvard University Press, Cambridge, MA.

SOPHOCLES, 1994b, Antigone, in H. Lloyd-Jones éd., The Women of Trachis, Philoctetes, Oedipus at Colonus, Harvard University Press, Cambridge, MA.

SOPHOCLES, 2004, Plays: Oedipus Tyrannus, R. Jebb. (sous la dir. de), P. Easterling (nouvelle éd.), Bristol Classical Press, London.

SOPHOCLES, 2002, King Oidipous, R. Blondell (sous la dir. de), Focus Classical Library, Newburyport, ME.

SOPHOCLES, 2006, Oedipus Rex, R. Dawe (sous la dir. de), (éd. révisée, éd. originale 1982), Cambridge University Press, Cambridge.

STEINER, R., 1994, In Vienna veritas... ? International Journal of Psychoanalysis, 75, 511-584.

VERNANT, J.-P., VIDAL-NAQUET, P., 1972, Mythe et tragédie en Grèce ancienne, Librairie François Maspéro, Paris.

VON TEPL, J., 1951, Der Ackermann aus Böhmen, M. Walshe (sous la dir. de), Duckworth, London, (éd. originale 1400.) 